\title{
Mechanism of Magnetic Pulse Wave Signal for Blood Pressure Measurement
}

\author{
Yang Zhang, Yibin Li, Xiaomeng Chen, Ning Deng \\ Institute of Microelectronics, Tsinghua University, Beijing, China \\ Email: yumaoqiuq@163.com, ningdeng@tsinghua.edu.cn
}

How to cite this paper: Zhang, Y., Li, Y.B., Chen, X.M. and Deng, N. (2016) Mechanism of Magnetic Pulse Wave Signal for Blood Pressure Measurement. J. Biomedical Science and Engineering, 9, 29-36. http://dx.doi.org/10.4236/jbise.2016.910B004

Received: March 27, 2016 Accepted: September 20, 2016

Published: September 23, 2016

\begin{abstract}
Continuous non-invasive blood pressure (BP) measurement can be realized by using pulse transit time (PTT) based on electrocardiogram (ECG) and pulse wave signal. Modulated magnetic signature of blood (MMSB) is a promising approach to obtain PTT. The origin of MMSB is critical to establish the relationship between MMSB and BP. In this paper, two possible origins of MMSB, blood disturbance mechanism and angular variation mechanism, are analyzed and verified through three control experiments under different conditions. The influence of blood velocity alteration and blood volume alteration on magnetic field is investigated though blood flow simulation system. It is found that MMSB comes mainly from the periodic blood flow while the perturbation caused by angular variation between sensitive axis of the magnetic sensor and geomagnetic field can be neglected. As to blood disturbance mechanism, the change of blood volume plays a decisive role while the effect of blood velocity alteration is negligible.
\end{abstract}

\section{Keywords}

Blood Pressure (BP), Modulated Magnetic Signature of Blood (MMSB), Pulse Transit Time (PTT)

\section{Introduction}

Blood pressure (BP) is one of the most important physiological parameters and it is closely related to human health. Normal BP can ensure normal blood circulation while high or low BP will be harmful to human health. Therefore, it is of great realistic significance to monitor BP real-timely. Currently, researchers mainly utilize pulse transit time (PTT) to achieve non-invasive continuous BP measurement. The method combined MMSB with electrocardiography (ECG) has been proposed to measure BP continuously [1] [2]. 
However, the origin of MMSB is not clear enough for practical applications. Most researchers believe that it is the blood flow below the sensor in a pulsating fashion that alters the magnetic field intensity near the sensor and produces MMSB [2]. To put it another way, some people believe that MMSB reflects the disturbance of magnetic field caused by blood flow [3] [4], while others argue that the slight movement of the magnetic sensor during experiment can also bring interference [5] [6]. Therefore, discussing the origin of MMSB is momentous for developing a real-time blood pressure monitoring system.

\section{Possible Origins of MMSB}

MMSB is usually obtained by a giant magneto-resistance (GMR) sensor above the radial artery in the bias magnetic field generated by a permanent magnet. Compared with conventional sensors, GMR sensor has many advantages such as high sensitivity, high reliability, wide measurement range and small volume. It can detect imperceptible changes of magnetic field and collect signals from human body readily. In previous studies, two possible origins have been proposed. One is called blood disturbance mechanism and the other is called angular variation mechanism. Two mechanisms are set forth below.

Periodic cardiac ejection makes some physical quantities related to blood flow change periodically such as blood velocity, vascular radius and blood pressure. Arterial blood and peripheral tissues have different permeability. Correspondingly, the induced magnetic fields by them are dissimilar. The magnetic field detected by GMR sensor changes as physical parameters change while peripheral tissue is almost constant compared with blood. So, it can be considered that it is periodic blood flow that makes surrounding magnetic field change periodically. This theory is called blood disturbance mechanism.

That pulse beats can bring greater interference to the measurement by GMR sensor because the GMR sensor experiences small movement caused by pulse beating in the experiment [5]. The angle between sensitive axis of magnetic sensor and geomagnetic field changes as the position of the sensor alters. Accordingly, it will introduce an extra interference to the detected signal. It is believed that the displacement of GMR sensor contributes to the MMSB besides the periodic blood flow. This theory is called angular variation mechanism. Some researchers even estimate the interference caused by the displacement approximately and draw some conclusions [5].

\section{Experimental Setup}

To get MMSB, a GMR sensor and a permanent magnet are essential. The MMSB acquisition system consists of GMR sensor, permanent magnet, A/D converter and back-end processing circuit as shown in Figure 1. The permanent magnet is placed about 2 centimeters away from the GMR sensor. This permanent magnet is only used to provide a bias magnet field so that the GMR sensor can work in the linear region. In other words, the magnet field generated by the permanent magnet at the position of GMR sensor is 
constant because both the permanent magnet and GMR sensor are fixed on the circuit board. In order to obtain a clear signal and input it into computer, back-end processing circuit and A/D converter are designed as well.

According to previous studies, MMSB may arise from periodic blood flow while it is still unclear whether blood velocity or blood volume plays a leading role. Hence, an extracorporeal blood flow simulation system is designed to verify which factor affects the magnetic field principally as shown in Figure 2 and Figure 3.

As the two pictures show, the system mainly includes adjustable speed pump, rubber tubes, a DC variable speed motor and a flume. Both the motor and the pump can be controlled by the governor which can alter the speed by regulating the voltage across them and display the percentage at the same time. The pump can provide power for blood circulation just like the human heart. The rubber tubes are used to complete the circulation instead of blood vessels. We alter the blood velocity by utilizing governor 2 and detect the magnetic field disturbed by blood flow at different speeds. The DC variable speed motor is used to generate periodic stress with an oval swivel in order to make the blood volume change periodically. Governor 1 is necessary to adjust the motor's

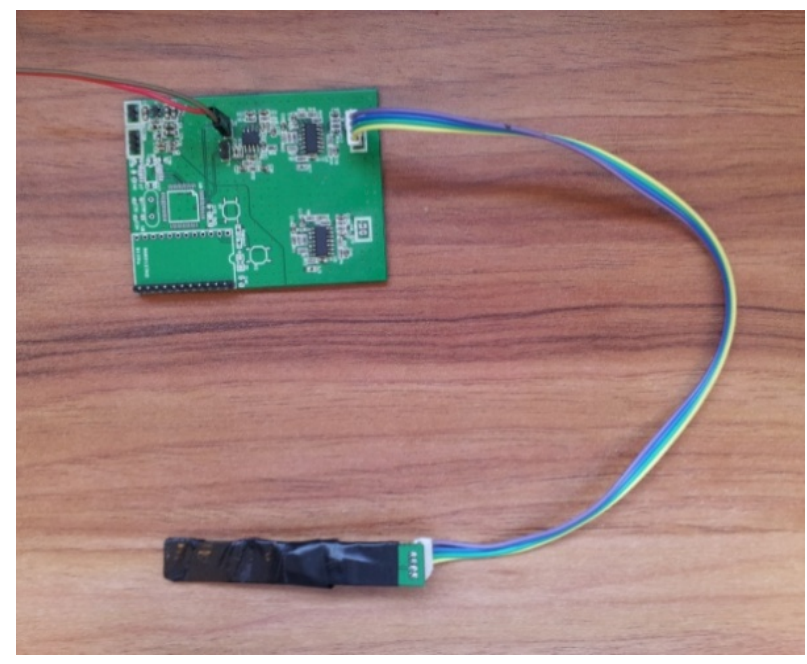

Figure 1. The MMSB acquisition system.

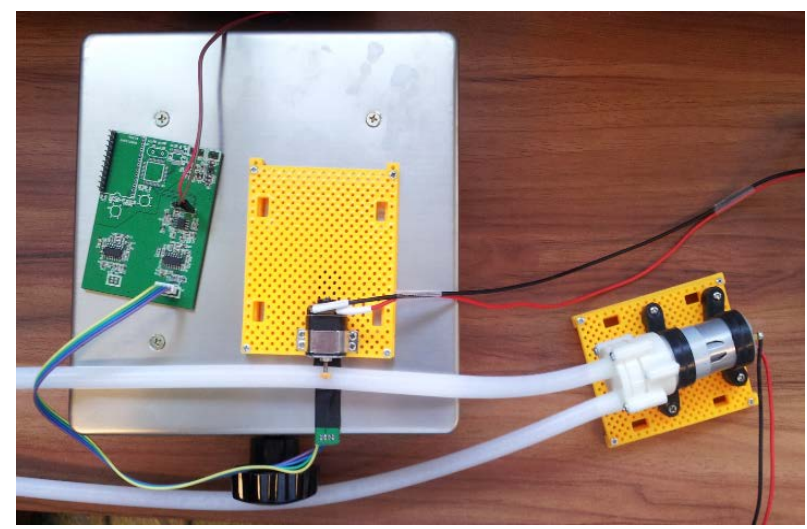

Figure 2. The extracorporeal blood flow simulation system. 


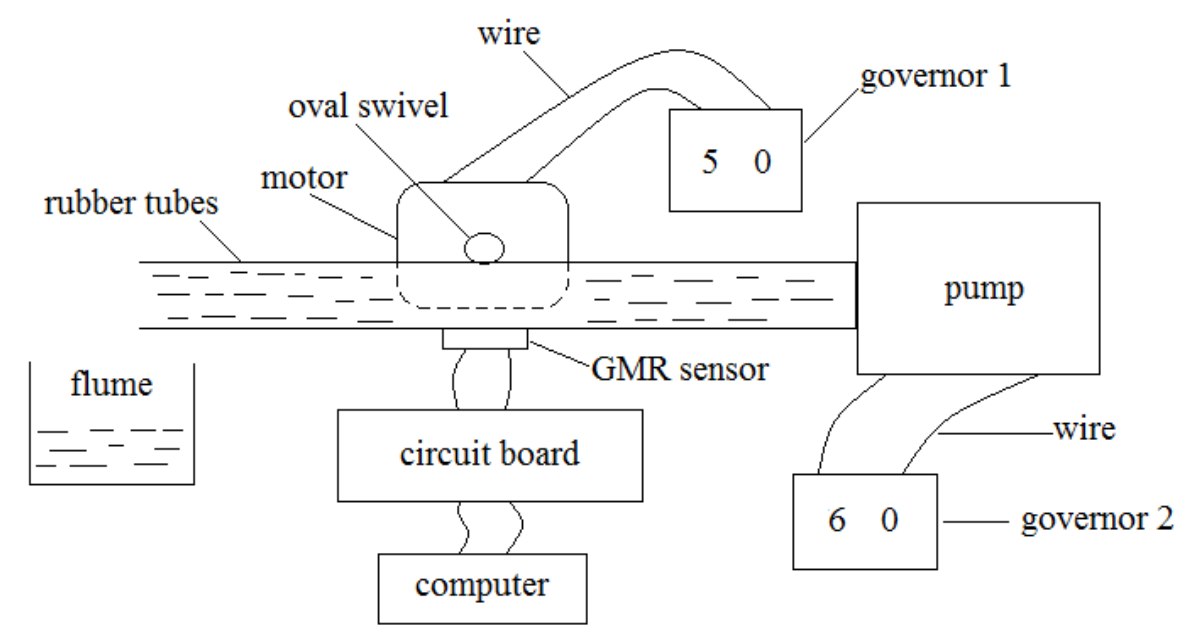

Figure 3. The schematic view of the simulation system.

speed and affect the pressure on the tube so that the blood volume can change as the human body.

We can change blood velocity and blood volume respectively by using the simulation system. Therefore, we can investigate the influence of blood velocity alteration and blood volume alteration on magnetic field respectively.

\section{Results and Discussions}

We collect MMSB from normal environment, magnetic shielded state and fixed sensor state. Normal environment means that the signal is gathered under normal circumstances and the circuit board with GMR sensor is held by the other hand. Magnetic shielded state is created by a magnetic shielding cylinder. The arm and the sensor are put into the magnetic shielding cylinder and the signal is detected under the condition of magnetic shielded. Fixed sensor state is produced by using an iron stand. The sensor is fixed with an iron clamp in this situation. Figure $\mathbf{4}$ shows the signals under different conditions.

As shown in Figure 4, MMSB signals under different conditions are extremely similar. In order to compare their size, we extract peaks of three MMSB signals respectively and list them in Table 1.

By comparing three signals' amplitude, it can be found that the amplitude of MMSB in normal environment is larger than that in magnetic shielded state while the amplitude of MMSB in magnetic shielded state is larger than that in fixed sensor state. The reason for this phenomenon is that different signals are caused by different mechanisms. It is no doubt that all the signals are affected by blood disturbance mechanism. However, angular variation mechanism does not work in the fixed sensor condition. In other words, MMSB of fixed sensor state is produced only by blood disturbance mechanism. Since the amplitudes of three signals are different, there are new factors affecting MMSB in normal environment and magnetic shielded state except blood disturbance mechanism. 

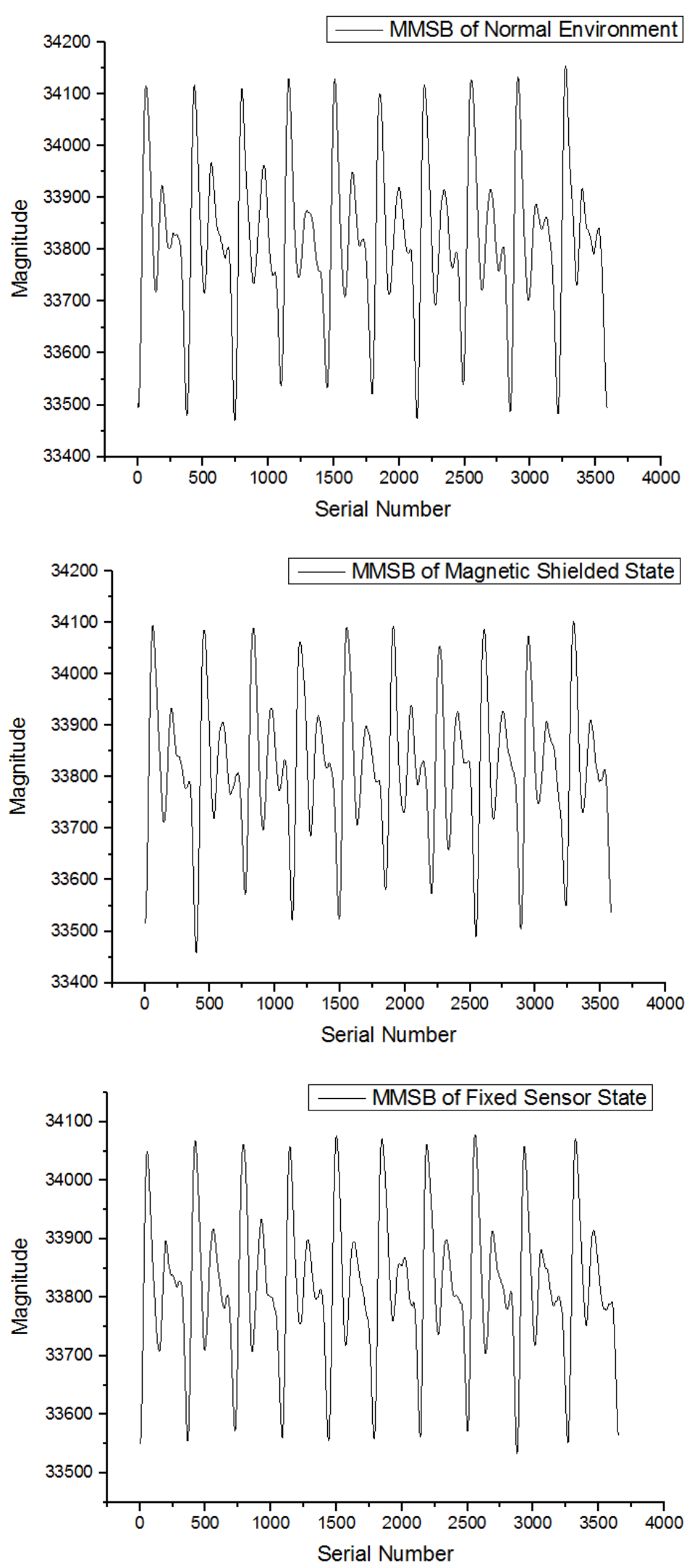

Figure 4. MMSB signals under different conditions. 
Table 1. Magnitude of three MMSB signals.

\begin{tabular}{cccc}
\hline Number & Normal Environment & Magnetic Shielded State & Fixed Sensor State \\
\hline 1 & 34116 & 34095 & 34049 \\
2 & 34118 & 34086 & 34068 \\
3 & 34111 & 34091 & 34063 \\
4 & 34130 & 34063 & 34058 \\
5 & 34129 & 34091 & 34077 \\
6 & 34101 & 34094 & 34071 \\
7 & 34118 & 34055 & 34062 \\
8 & 34128 & 34088 & 34079 \\
9 & 34133 & 34074 & 34058 \\
10 & 34155 & 34102 & 34071 \\
Average & 34123.9 & 34083.9 & 34065.6 \\
\hline
\end{tabular}

It is easy to be accepted that MMSB of normal environment is caused by blood disturbance mechanism and angular variation mechanism at the same time. The geomagnetic field in the magnetic shielding cylinder is almost zero. However, there is a permanent magnet in MMSB acquisition system and the permanent magnet can magnetize the magnetic shielding cylinder in a very short time. Thus, when collecting MMSB in magnetic shielding cylinder, magnetized cylinder is equivalent to a small magnet and it can bring interference though angular variation mechanism. To put it another way, MMSB of magnetic shielded state is also caused by two mechanisms at the same time. Since the magnetic field generated by magnetized cylinder is weaker than geomagnetic field, the influence of angular variation mechanism in magnetic shielded state is smaller than that in normal environment.

Another possible factor affecting MMSB of fixed sensor state is measurement error. It is probable to lead to a slight increase in the distance between the sensor and blood owing to the fixed sensor compared with the other two conditions while the measurement error mainly results from the longer distance. These explain thoroughly why the amplitude of MMSB in normal environment is maximal while the amplitude of MMSB in fixed sensor state is minimal. Although MMSB of normal environment and MMSB of fixed sensor state are caused by different mechanisms, the difference value of their amplitude is 58.3. The value of 58.3 takes up only 8.4 percent of the whole waveform. Considering the error caused by fixed sensor, angular variation mechanism's contribution to MMSB is less than $8.4 \%$. Therefore, we can conclude that MMSB is caused mainly by blood disturbance mechanism.

Another set of experiments is mainly used to testify the effect of blood velocity alteration and blood volume alteration on magnetic field. Firstly, we make the liquid flow in the rubber tube in a steady state and measure the magnetic induction under the tube at the speed of $0 \mathrm{~m} / \mathrm{s}, 0.4 \mathrm{~m} / \mathrm{s}$ and $0.8 \mathrm{~m} / \mathrm{s}$. The speed of the liquid can be regulated by 
governor 2. It is calculated by a number of parameters such as the tube's diameter, the rated speed of the motor and the percentage of governor 2 .

Table 2 lists magnetic inductions in the same position at various speeds and the unit of magnetic induction is milligauss.

As shown in Table 2, there is little difference among magnetic inductions at various speeds. Moreover, the difference of these data is mainly from the noise and the experimenter's posture.

Then, we use the DC variable speed motor with an oval swivel to change the blood volume periodically. One of the most important criteria of the accuracy of the artificial signal is the MMSB's period. Therefore, the signal's period is modulated close to the human body by applying governor 1 . The change of magnetic field is shown in Figure 5.

Table 2. Magnetic inductions at various speeds.

\begin{tabular}{cccc}
\hline Number & $0 \mathrm{~m} / \mathrm{s}$ & $0.4 \mathrm{~m} / \mathrm{s}$ & $0.8 \mathrm{~m} / \mathrm{s}$ \\
\hline 1 & 19.52 & 19.58 & 19.51 \\
2 & 19.56 & 19.54 & 19.47 \\
3 & 19.59 & 19.56 & 19.53 \\
4 & 19.53 & 19.52 & 19.60 \\
5 & 19.55 & 19.50 & 19.55 \\
6 & 19.46 & 19.59 & 19.58 \\
7 & 19.48 & 19.53 & 19.50 \\
8 & 19.51 & 19.48 & 19.47 \\
9 & 19.56 & 19.51 & 19.56 \\
10 & 19.54 & 19.53 & 19.533 \\
Average & & & 19.531
\end{tabular}

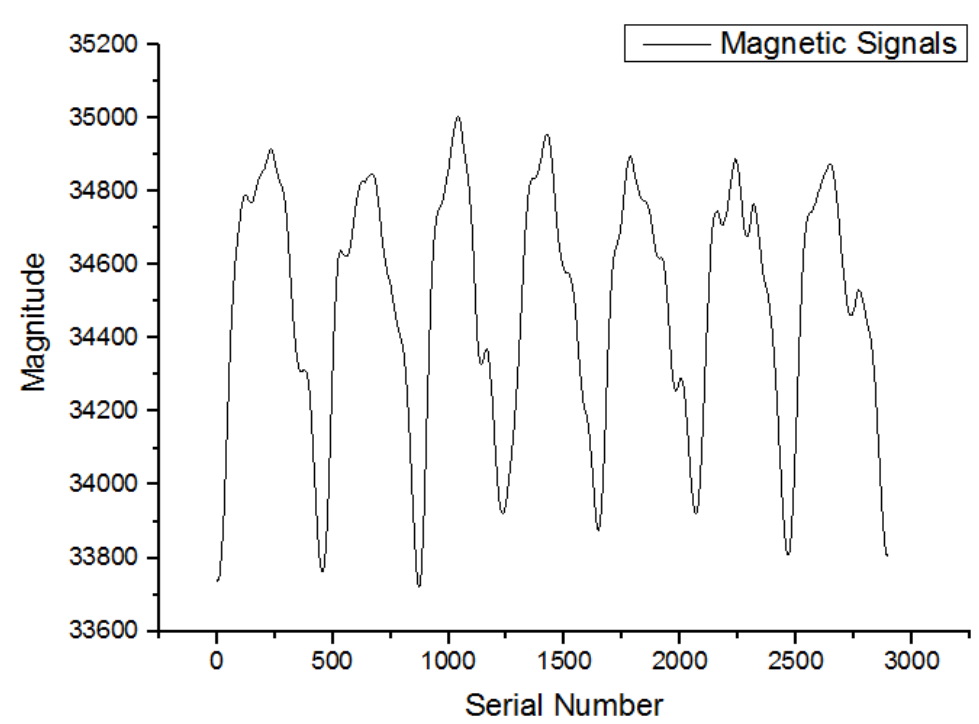

Figure 5. Magnetic signals by changing volume periodically. 
As shown in Figure 5, the change of magnetic field is extreme obvious. The difference value between maximum point and minimum point is about 1160 and it is almost twice as much as MMSB. So, we can conclude that blood volume alteration plays a crucial role in blood disturbance mechanism while the effect of blood velocity alteration can be disregarded.

\section{Conclusion}

In this paper, two possible origins of MMSB are investigated. Theoretically, both blood disturbance mechanism and angular variation mechanism contribute to MMSB. For practical applications, MMSB is determined by blood disturbance mechanism rather than angular variation mechanism. The angular variation between sensitive axis of magnetic sensor and geomagnetic field can be neglected. In other words, it is blood volume alteration that makes magnetic field change regularly while blood velocity alteration has little effect on magnetic field. This conclusion can be used for blood pressure measurement based on MMSB.

\section{References}

[1] Phua, C.T. and Lissorgues, G. (2009) Measurement of Blood Pressure Using Magnetic Method of Blood Pulse Acquisition. Proceedings of the 2009 IEEE 3 rd International Conference on Nano/Molecular Medicine and Engineering, Taiwan, October 2009, 112-115. http://dx.doi.org/10.1109/NANOMED.2009.5559106

[2] Joseph, J. and Jayashankar, V. (2010) Magnetic Sensor for Non-Invasive Detection of Blood Pulse and Estimation of Arterial Compliance. IEEE EMBS Conference on Biomedical Engineering \& Sciences, Kuala Lumpur, Malaysia, 30 December 2010, 170-175. http://dx.doi.org/10.1109/iecbes.2010.5742222

[3] Phua, C.T., Lissorgues, G. and Mercier, B. (2008) Non-Invasive Acquisition of Blood Pulse using Magnetic Disturbance Technique. Proceedings of 23rd International Conference of Biomedical Engineering, 786-789.

[4] Joseph, J., Venkataraman, J. and Jagadeesh, V.K. (2011) Non-Invasive Estimation of Arterial Compliance. IEEE International Instrumentation \& Measurement Technology Conference, $480-484$

[5] Li, Y.B., Gao, Y.Y. and Deng, N. (2013) Mechanism of Cuff-Less Blood Pressure Measurement Using MMSB. The 7 th International Conference on Bioinformatics and Biomedical Engineering, 123-125.

[6] Sinatra, F.L. (2010) Understanding the Interaction between Blood Flow and an Applied Magnetic Field. University of South Florida. 
Submit or recommend next manuscript to SCIRP and we will provide best service for you:

Accepting pre-submission inquiries through Email, Facebook, LinkedIn, Twitter, etc.

A wide selection of journals (inclusive of 9 subjects, more than 200 journals)

Providing 24-hour high-quality service

User-friendly online submission system

Fair and swift peer-review system

Efficient typesetting and proofreading procedure

Display of the result of downloads and visits, as well as the number of cited articles

Maximum dissemination of your research work

Submit your manuscript at: http://papersubmission.scirp.org/

Or contact jbise@scirp.org 